\title{
Fuzheng Qingjie granules potentiate the anticancer effect of cyclophosphamide by regulating cellular immune function and inducing apoptosis in Hepatoma 22 tumor-bearing mice
}

\author{
XU-ZHENG CHEN $^{1}$, ZHI-YUN CAO ${ }^{1}$, YOU-QUAN ZHANG ${ }^{2}$, JIN-NONG LI ${ }^{3}$, LIAN-MING LIAO ${ }^{1}$ and JIAN DU ${ }^{1}$ \\ ${ }^{1}$ Center of Oncology, Academy of Integrative Medicine, Fujian University of Traditional Chinese Medicine, \\ Fuzhou, Fujian 350122; ${ }^{2}$ Department of Clinical Laboratory, The Second Affiliated Hospital, Fujian University \\ of Traditional Chinese Medicine, Fuzhou, Fujian 350003; ${ }^{3}$ Department of Pharmacognosy, College of \\ Pharmacy, Fujian University of Traditional Chinese Medicine, Fuzhou, Fujian 350122, P.R. China
}

Received July 20, 2015; Accepted January 6, 2017

DOI: $10.3892 / 01.2017 .5849$

\begin{abstract}
Fuzheng Qingjie (FZQJ) is a polyherbal Chinese medicine that has previously been implemented as an adjuvant therapy for gastrointestinal cancer. The present study investigated whether FZQJ is able to potentiate the anticancer effect of cyclophosphamide (CTX). Hepatoma 22 tumor-bearing mice were randomly divided into a vehicle group, CTX group, FZQJ group and combination (CTX+FZQJ) group. In addition, untreated mice without $\mathrm{H} 22$ cells served as blank controls. Seven days post-treatment, the mice were sacrificed and the tumors were weighed. Blood cells were evaluated using an automatic hemocytometer analyzer and flow cytometer. The expression levels of interleukin (IL)-2 and tumor necrosis factor (TNF)- $\alpha$ were evaluated using a radioimmunoassay. Apoptotic cells were observed using a terminal deoxynucleotidyl transferase dUTP nick-end labeling assay. Alanine transaminase, aspartate aminotransferase, blood urea nitrogen and creatinine were examined using an automatic biochemical analyzer. The results demonstrated that the tumor inhibitory
\end{abstract}

Correspondence to: Dr Lian-Ming Liao or Professor Jian Du, Center of Oncology, Academy of Integrative Medicine, Fujian University of Traditional Chinese Medicine, 1 Qiuyang Road, Fuzhou, Fujian 350122, P.R. China

E-mail:1lm@fjtcm.edu.cn

E-mail: dujian@fjtcm.edu.cn

Abbreviations: HCC, Hepatocellular carcinoma; TCM, Traditional Chinese medicine; FZQJ, Fuzheng Qingjie; CTX, cyclophosphamide; NK cell, natural killer cell; IL, interleukin; TNF, tumor necrosis factor; RT-PCR, reverse transcription-polymerase chain reaction; HRP, horseradish peroxidase; TUNEL, terminal deoxynucleotidyl transferase dUTP nick-end labeling; $\mathrm{H} \& \mathrm{E}$, hematoxylin and eosin; WBC, white blood cells; RBC, red blood cells; PLT, platelet; Hb, hemoglobin

Key words: Fuzheng Qingjie granules, hepatoma, apoptosis, cyclophosphamide rate and apoptosis index were higher in the combination group, compared with those in the CTX group. Notably, FZQJ was able to alleviate CTX-induced decreases in the numbers of white blood cells and platelets, $\mathrm{CD}^{+}$and $\mathrm{CD}^{+} \mathrm{T}$ lymphocyte subsets, and the concentration of hemoglobin, body weight and thymus index, and increase serum TNF- $\alpha$ and IL-2 levels without overt hepatorenal toxicity. These results suggest that FZQJ granules may enhance the anticancer effect of CTX, in addition to alleviating the side effects.

\section{Introduction}

Hepatocellular carcinoma (HCC) is one of the most frequent causes of cancer-associated mortality globally due to a high incidence and poor prognosis (1). This is particularly high in China due to the $10 \%$ hepatitis $\mathrm{B}$ virus infection rate in the general population (2). The majority of patients with HCC are diagnosed at intermediate or advanced stages of disease, excluding them from potentially curative treatment options, including resection, local ablation or liver transplantation (3). For intermediate or advanced HCC, chemotherapy is not routinely used as, in addition to severe adverse reactions, including myelosuppression, nausea and vomiting, the response rate is $\sim 10-20 \%$ (4). Therefore, there is an urgent requirement to improve the efficacy of chemotherapy and simultaneously decrease the associated adverse reactions.

Traditional Chinese medicine (TCM) emphasizes the importance of inhibiting tumor growth and alleviating the adverse reactions of chemotherapy and/or radiotherapy in order to improve patient quality of life (5). TCM physicians often use Fuzheng Guben herbs and Qingre Jiedu herbs during cancer treatment (6). Fuzheng Guben herbs, including Ganodorma lucidum, Astragalus membranaceus, Ginseng species and Fructus lycii, have been observed to strengthen the immune response through the activation of $\mathrm{T}$ and $\mathrm{B}$ lymphocytes, macrophages, natural killer (NK) cells and dendritic cells, and promoting the production of cytokines, including interleukins (IL), tumor necrosis factors (TNF) and interferon (7). Additionally, these herbs have been demonstrated to protect bone marrow from cyclophosphamide (CTX) and 
cytosine arabinoside, and prevent a chemotherapy-induced decrease in white blood cells (WBC), red blood cells (RBC) and platelets (PLT) in the peripheral blood (8). According to TCM, heat is considered an important causative factor in $\mathrm{HCC}$, and Qingre Jiedu has been administered to clear heat and detoxify the body (6). Previous pharmacological studies have demonstrated that Qingre Jiedu herbs (including Hedyotis diffusa Willd, Prunella vulgaris, Lobelia chinensis Lour and Sophora flavescens) contain anthraquinones, polysaccharides, flavonoids, alkaloids and triterpenoids that are able to inhibit tumor cell proliferation, induce cell apoptosis and suppress angiogenesis (9-11). Matrine from Sophora flavescens is able to induce the apoptosis of HepG2 cells via the upregulation of tumor protein 53, B cell lymphoma-2 (Bcl-2)-associated X protein (Bax) and Fas, and the downregulation of Bcl-2 and c-Myc (12).

Fuzheng Qingjie granules (FZQJ) are composed of Fuzheng Guben and Qingre Jiedu herbs (13). The four Fuzheng Guben herbs are Astragalus membranaceus, Ligustrum lucidum, Ganoderma lucidum and Rhizoma dioscoreae, and the two Qingre Jiedu herbs are Hedyotis diffusa Willd and Prunella vulgaris (13). FZQJ granules are used to treat the symptoms that are commonly observed post-chemotherapy or radiotherapy, including thirst, night sweats, constipation, insomnia, loss of appetite and weakness. A previous study demonstrated that FZQJ may induce apoptosis of HepG2 cells via activating p38 mitogen-activated protein kinases (MAPKs) and inducing mitochondria-dependent apoptosis (13).

CTX is biotransformed principally in the liver to active alkylating metabolites, which cross-link tumor cell DNA (14) in order to interfere with the growth of susceptible rapidly proliferating malignant cells. CTX is used in combination with other antineoplastic drugs to treat a variety of susceptible malignancies, including lymphoma (15) and myeloma (16), ovarian (17), nasopharyngeal (18) and liver cancer (19). However, CTX is also associated with severe toxicities, including diarrhea, nausea, vomiting, bone marrow suppression, haemorrhagic cystitis, fatigue, night sweat, hair loss, immunosuppression and impaired hepatic and renal function (20).

The present study investigated whether FZQJ is able to potentiate the anticancer effects of CTX in hepatoma 22 (H22) tumor-bearing mice, and potentially alleviate CTX-associated toxicities.

\section{Materials and methods}

Preparation of FZQJ decoction. FZQJ was manufactured and provided by the Department of Pharmacy, The Second Affiliated Hospital, Fujian University of Traditional Chinese Medicine (Fuzhou, China). FZQJ granules were dissolved in distilled water to produce a solution with a final concentration of $0.3 \mathrm{~g} / \mathrm{ml}$, which was stored at $4^{\circ} \mathrm{C}$ until use.

Mouse xenograft experiments. A total of 50 male specific pathogen free Institute of Cancer Research mice (6-weeks-old, 22-25 g) were purchased from Guangdong Animal Center (Guangzhou, China). All animals lived in a light/dark cycle. Food and tap water were available ad libitum. The room temperature (RT) was maintained at $23 \pm 2^{\circ} \mathrm{C}$ and humidity was approximately $60 \%$. The mice were inoculated subcutaneously on the right side of theirs back with $5 \times 10^{6}$ H22 cells in Matrigel/Dulbecco's Modified Eagle's medium with gentamycin (BD Biosciences, Franklin Lakes, NJ, USA). H22 cells were purchased from the Shanghai Institute of Life Science (Chinese Academy of Sciences, Shanghai, China) and cultured in RPMI-1640 culture medium (Gibco; Thermo Fisher Scientific, Inc., Waltham, MA, USA) at $37^{\circ} \mathrm{C}$ with $5 \% \mathrm{CO}_{2}$. Mice were randomly divided into 4 groups ( $\mathrm{n}=10$ per group) as follows: Vehicle group (oral distilled water), CTX group (Baxter Oncology GmbH, Halle, Saxony, Germany, intraperitoneally; $40 \mathrm{mg} / \mathrm{kg} /$ day), FZQJ group (oral; $6 \mathrm{~g} / \mathrm{kg} /$ day) and combination group (CTX $40 \mathrm{mg} / \mathrm{kg} /$ day intraperitoneally plus FZQJ $6 \mathrm{~g} / \mathrm{kg} /$ day orally). Mice in the vehicle control group were inoculated with $\mathrm{H} 22$ cells and administered distilled water orally. In addition, another ten mice without $\mathrm{H} 22$ cells served as the blank group. At day seven, all the mice were sacrificed by cervical dislocation and peripheral blood was collected by ocular enucleation. The tumors were dissected by a surgical operation and weighed.

Thymus index. The thymus was collected from the mice by a surgical operation and weighed. The thymus index was calculated according to the following formula: Thymus index $(\mathrm{mg} / 10 \mathrm{~g})=$ thymus weight $(\mathrm{mg}) /$ body weight $(\mathrm{g}) \times 10$.

Routine blood analysis. WBC, lymphocyte (LY) and RBC cell numbers as well as PLT and hemoglobin $(\mathrm{Hb})$ concentrations, were examined with EDTA-K2 anticoagulated whole blood using an automatic hemocytometer (Sysmex Corporation, Kobe, Japan). This process was performed $\geq 3$ times for each blood sample.

$T$ and NK immune cells. Anticoagulated whole blood was stained with a fluorescein isothiocyanate-conjugated cluster of differentiation (CD) 3 monoclonal antibody (mAb; cat. no. 100203; dilution, 1:200, BioLegend, Inc., San Diego, CA, USA), in combination with a phycoerythrin (PE)-conjugated CD8a mAb (cat. no. 100707; dilution, 1:80; BioLegend, Inc.), or PE-conjugated CD4 mAb (cat. no. 100509; dilution, 1:200; BioLegend, Inc.) or PE-conjugated CD49b mAb (cat. no., 108907; dilution, 1:80; BioLegend, Inc.), incubated at RT for 15 min in the dark and subsequently lysed using BD FACS ${ }^{\mathrm{TM}}$ lysing solution (BD Biosciences). A FACSCalibur ${ }^{\mathrm{TM}}$ flow cytometer with CellQuest software (version 5.1; BD Biosciences) was utilized to analyze the percentage of $\mathrm{CD}^{+} \mathrm{T}$ cells, $\mathrm{CD}^{+}{ }^{+} \mathrm{T}$ cells, $\mathrm{CD} 8^{+} \mathrm{T}$ cells and NK cells present. Absolute counts of $\mathrm{CD}^{+} \mathrm{T}$ cells, $\mathrm{CD} 4^{+} \mathrm{T}$ cells, $\mathrm{CD} 8^{+} \mathrm{T}$ cells and $\mathrm{NK}$ cells were calculated according to the following formula: Absolute cell number $=$ the percentage of cells $\mathrm{x}$ the number of LYs. All experiments were performed $\geq 3$ times.

Cytokine assays. The serum levels of IL-2 and TNF- $\alpha$ in H22 tumor-bearing mice were determined using a $\gamma$ radioimmunoassay counter (Dongya Immunological Technique Institute, Beijing, China), with ${ }^{125} \mathrm{I}-\mathrm{IL}-2$ and ${ }^{125} \mathrm{I}-\mathrm{TNF}-\alpha$ radioimmunoassay kits according to the protocol of the manufacturer (Dongya Immunological Technique Institute).

Hepatic and renal functions of $\mathrm{H} 22$ tumor-bearing mice. To determine the safety of FZQJ, the serum levels of alanine 

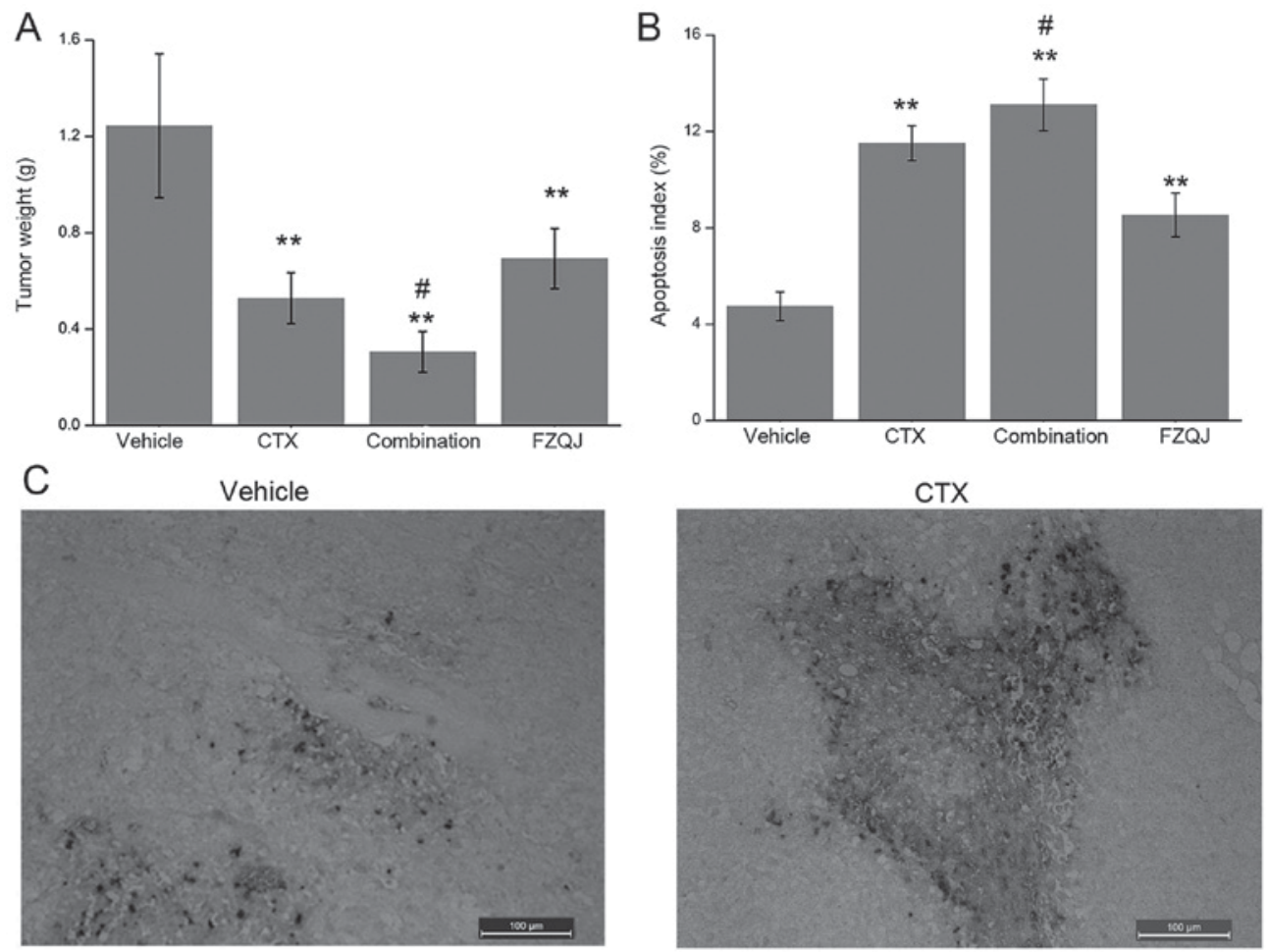

Combination

FZQJ
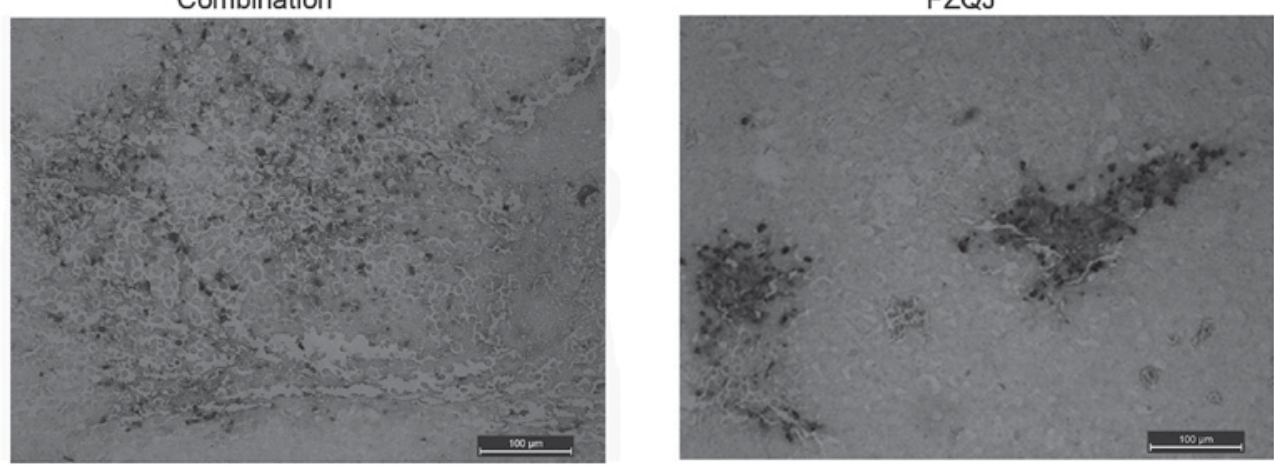

Figure 1. Comparisons of tumor weight and apoptosis index. (A) Comparison of tumor weight between the vehicle, CTX, combination and FZQJ groups. "* $\mathrm{P}<0.01$, compared with the vehicle group; ${ }^{*} \mathrm{P}<0.05$, compared with the CTX group. (B) Apoptosis index was calculated by dividing the number of TUNEL-positive cells by the total number of cells in the field. ${ }^{* *} \mathrm{P}<0.01$, compared with the vehicle group; ${ }^{\#} \mathrm{P}<0.05$, compared with the CTX group. (C) Representative images of TUNEL assays (magnification, x 200; scale bar, $100 \mu \mathrm{m}$ ). TUNEL-positive cells are stained dark brown. CTX, cyclophosphamide; FZQJ, Fuzheng Qingjie; TUNEL, terminal deoxynucleotidyl transferase dUTP nick-end labeling.

transaminase (ALT), aspartate aminotransferase (AST), blood urea nitrogen (BUN) and creatinine (CRE) were assessed according to the manufacturer's protocol with a FLEX mode automatic biochemical analyzer (TBA-120FR, Toshiba, Kawasaki, Japan).

Terminal deoxynucleotidyl transferase dUTP nick-end labeling (TUNEL) assay. The tumor tissues were fixed in $4 \%$ paraformaldehyde at RT for $36 \mathrm{~h}$. Following dehydration, the fixed tissues were embedded in paraffin. The samples were sectioned at thickness of $5 \mu \mathrm{m}$. Subsequently, the sections were deparaffinized with $\geq 99 \%$ xylene baths at RT, 10 min each, and then rehydrated in graded ethanol solutions of 100 , 95,70 , and $50 \%(\mathrm{v} / \mathrm{v})$. The apoptotic cells of the tumors were detected using a TUNEL assay, according to the manufacturer's protocol (Fuhzou Maixin Biotech Co., Ltd., Fuzhou, China). TUNEL-positivity indicates that the cells exhibit the DNA damage that results from apoptotic cascades (21).
These cells possessed a pyknotic nucleus with dark brown staining, and were counted in 10 random fields at x200 magnification. The apoptotic index was reported as the number of TUNEL-positive cells/total number of cells scored.

Statistical analysis. Statistical analysis was performed using SPSS version 18.0 (SPSS, Inc., Chicago, IL, USA). Data were presented as the mean \pm standard deviation. For multiple comparisons, the data were analyzed using one-way analysis of variance. $\mathrm{P}<0.05$ was considered to indicate a statistically significant difference.

\section{Results}

FZQJ potentiates the anticancer effect of CTX. To evaluate whether FZQJ is able to potentiate the anticancer effect of CTX, the tumor xenograft weight of each mouse was examined at seven days post-treatment. As presented in Fig. 1A, 

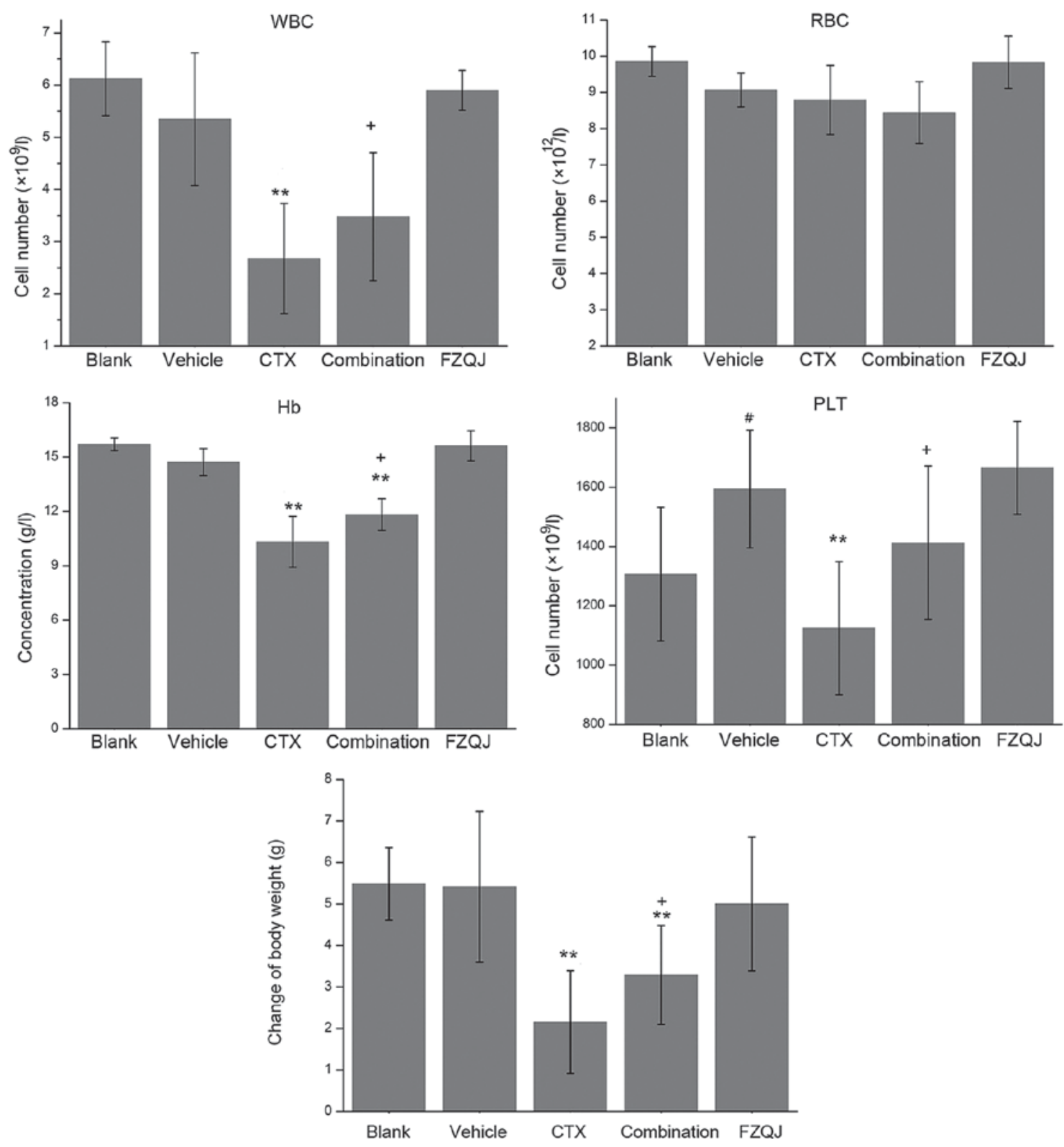

Figure 2. Comparison of blood cell counts in peripheral blood and mouse body weight. Compared with the blank group, PLT counts in the vehicle group significantly increased $\left({ }^{\#} \mathrm{P}<0.05\right)$. Compared with the vehicle group, WBC and PLT counts, Hb concentration and body weight in the CTX group significantly decreased $\left({ }^{* *} \mathrm{P}<0.01\right)$. These effects were alleviated when FZQJ was administered in combination with $\mathrm{CTX}\left({ }^{+} \mathrm{P}<0.05\right)$. CTX, cyclophosphamide; FZQJ, Fuzheng Qingjie; WBC, white blood cell; RBC, red blood cell; Hb, hemoglobin; PLT, platelet.

compared with the vehicle group, tumor weight significantly decreased in all three groups $(\mathrm{P}<0.01)$. No significant difference was observed between the CTX group and FZQJ group $(\mathrm{P}=0.156)$. Notably, when these drugs were administered simultaneously, the inhibitory rate was higher, compared with CTX alone $(\mathrm{P}=0.027)$. Therefore, FZQJ may potentiate the anticancer effect of CTX.

In addition, TUNEL assays were performed in order to assess the apoptotic cells in $\mathrm{H} 22$ neoplastic tissue (Fig. 1B), in which the TUNEL-positive cells were stained dark brown. The apoptosis index in all three treated groups was significantly higher, compared with the vehicle group $(\mathrm{P}<0.01$; Fig. 1C). Compared with the CTX group, the apoptosis percentage was higher in the combination group $(\mathrm{P}=0.049)$. These data demonstrated that FZQJ may be able to potentiate the anticancer effect of CTX through the induction of apoptosis.
FZQJ alleviates CTX-induced peripheral blood cell and body weight decreases. To evaluate whether FZQJ is able to alleviate the adverse effects of CTX, the numbers of WBCs, RBCs, PLTs, the concentration of $\mathrm{Hb}$ in the peripheral blood and the body weight of the mice over the course of treatment were examined. As presented in Fig. 2, PLT counts were higher in the vehicle group, compared with the blank group $(\mathrm{P}=0.048)$. The levels of WBCs, RBCs, PLTs and Hb were similar between the vehicle group and the FZQJ group $(5.34 \pm 1.27) \times 10^{9} / 1$ vs $(5.90 \pm 0.38) \times 10^{9} / 1,(9.07 \pm 0.47) \times 10^{12} / 1$ vs. $(9.83 \pm 0.72) \times 10^{12} / 1$, $(1594.00 \pm 198.66) \times 10^{9} / 1$ vs. $(1665.60 \pm 156.87) \times 10^{9} / 1$, $14.72 \pm 0.74$ vs. $15.62 \pm 0.83 \mathrm{~g} / \mathrm{l}$, respectively, indicating that FZQJ granules are non-toxic to bone marrow. As hypothesized, CTX markedly decreased the WBC and PLT counts, as well as the concentration of $\mathrm{Hb}$, due to its induction of bone marrow suppression. By contrast, when CTX and FZQJ 

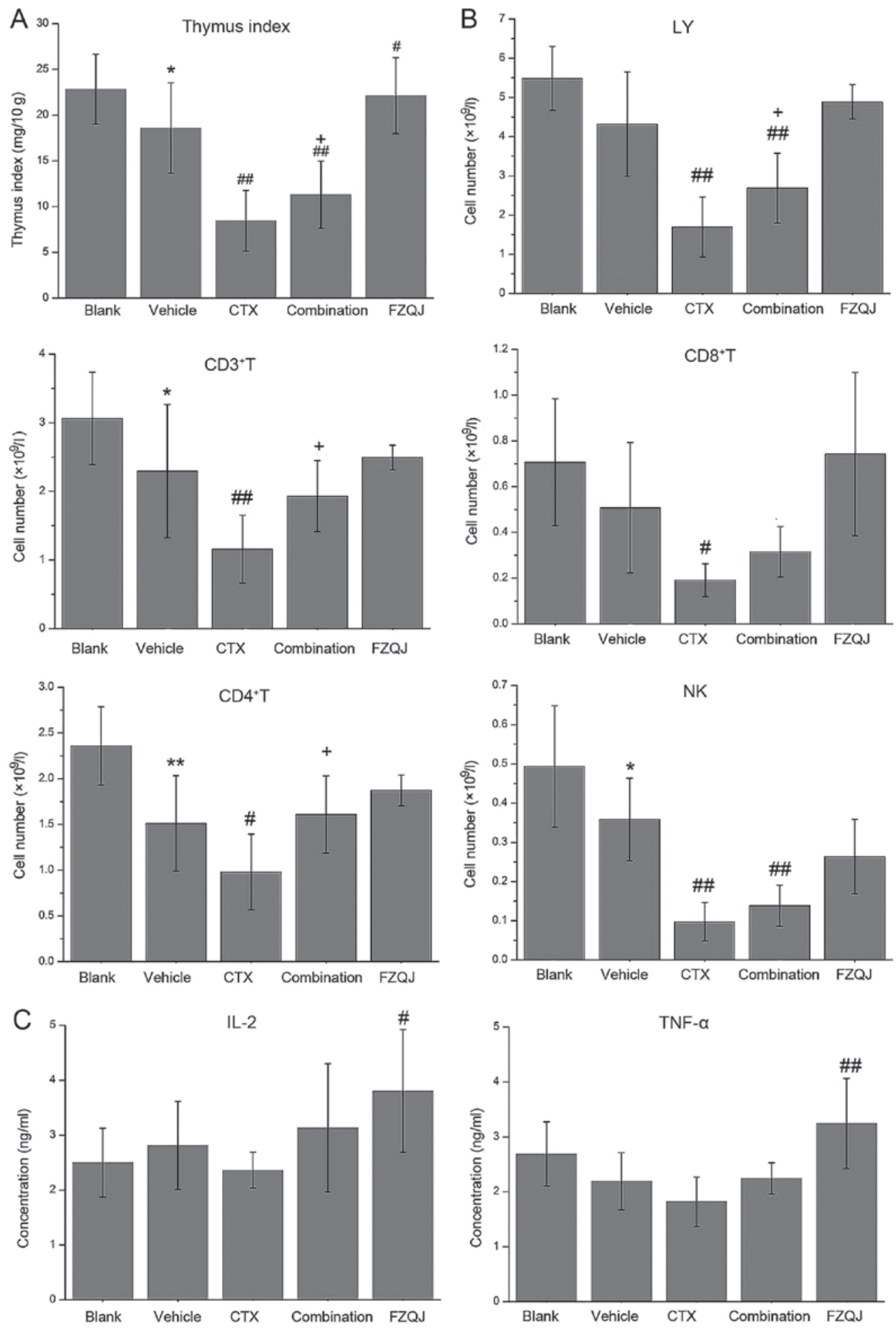

Figure 3. Comparison of immune function in H22 tumor-bearing mice. (A) Comparison of thymus index. (B) Comparison of LY, CD3+ T, CD4+ T, CD8+ T and NK cell counts. (C) Comparison of expression levels of IL- 2 and TNF- $\alpha$ in sera as evaluated by a radioimmunoassay. " $\mathrm{P}<0.05$ and ${ }^{* *} \mathrm{P}<0.01$, compared with the blank group; ${ }^{\#} \mathrm{P}<0.05$ and ${ }^{\# "} \mathrm{P}<0.01$, compared with the vehicle group; ${ }^{+} \mathrm{P}<0.05$, compared with the CTX group. CTX, cyclophosphamide; FZQJ, Fuzheng Qingjie; LY, lymphocytes; CD, cluster of differentiation; IL, interleukin; TNF, tumor necrosis factor; NK, natural killer.

were simultaneously administered, these parameters were all significantly improved (WBCs, $\mathrm{P}=0.048$; PLTs, $\mathrm{P}=0.047$; Hb, $\mathrm{P}=0.016$ ). Similarly, a significant difference was not observed with respect to the body weight of the mice between the vehicle group and the FZQJ group. And CTX notably induced body weight loss $(\mathrm{P}<0.01)$. The $\mathrm{CTX}$-induced body weight decrease was prevented by FZQJ $(\mathrm{P}=0.050)$.
FZQJ improves immune function in H22 tumor-bearing mice. To evaluate the effect of FZQJ on the immune function of $\mathrm{H} 22$ tumor-bearing mice, the subpopulations of lymphocyte cells, thymus index, serum IL-2 and TNF- $\alpha$ levels were examined. As presented in Fig. 3A, the thymus index in the H22 tumor-bearing mice was significantly decreased, compared with the blank group $(\mathrm{P}=0.021)$. The thymus index was further 
Table I. Hepatic and renal functions of H22-tumor bearing mice.

\begin{tabular}{lcccc}
\hline Group & ALT, IU/l & AST, IU/l & BUN, mmol/l & CRE, $\mu \mathrm{mol} / \mathrm{l}$ \\
\hline Normal & $45.25 \pm 9.36$ & $223.75 \pm 23.14$ & $7.73 \pm 1.52$ & $18.53 \pm 2.10$ \\
Vehicle & $50.20 \pm 7.53$ & $220.80 \pm 30.36$ & $7.70 \pm 1.23$ & $19.38 \pm 3.08$ \\
CTX & $49.20 \pm 9.93$ & $429.00 \pm 55.84^{\mathrm{a}}$ & $7.48 \pm 1.41$ & $16.68 \pm 2.91$ \\
Combination (CTX+FZQJ) & $53.00 \pm 9.56$ & $433.75 \pm 54.99^{\mathrm{a}}$ & $7.02 \pm 0.99$ & $18.30 \pm 2.79$ \\
FZQJ & $52.00 \pm 8.37$ & $225.00 \pm 34.73$ & $6.94 \pm 1.34$ & $17.28 \pm 2.05$ \\
\hline
\end{tabular}

${ }^{\mathrm{a}} \mathrm{P}<0.01$ vs. the vehicle group. All data presented as mean \pm standard deviation. ALT, alanine transaminase; AST, aspartate aminotransferase; BUN, blood urea nitrogen; CRE, creatinine; CTX, cyclophosphamide; FZQJ, Fuzheng Qingjie; IU/l, international units per liter.

decreased when CTX was administered $(\mathrm{P}=0.000)$, whilst FZQJ was able to increase the thymus index of non-treated $(\mathrm{P}=0.030)$ and $\mathrm{CTX}$-treated $\mathrm{H} 22$ tumor-bearing mice $(\mathrm{P}=0.049)$.

In addition, the absolute counts of $\mathrm{CD}^{+} \mathrm{T}, \mathrm{CD} 4^{+} \mathrm{T}$ and NK cells in the vehicle group were notably lower, compared with those in the blank group $(\mathrm{P}=0.039,0.004,0.045$ respectively; Fig. 3B), an effect that tended to be reversed by the administration of FZQJ. As hypothesized, CTX markedly decreased the numbers of $\mathrm{LYs}, \mathrm{CD}^{+} \mathrm{T}, \mathrm{CD}^{+} \mathrm{T}, \mathrm{CD} 8^{+} \mathrm{T}$ and NK cells (Fig. 3B). The addition of FZQJ to CTX increased the numbers of $\mathrm{LYs}, \mathrm{CD}^{+} \mathrm{T}$ and $\mathrm{CD} 4^{+} \mathrm{T}$ cells. As presented in Fig. 3C, it was observed that the levels of IL-2 and TNF- $\alpha$ were highest in the FZQJ groups $(\mathrm{P}=0.049$ and 0.006 respectively, compared with the vehicle group), and IL-2 and TNF- $\alpha$ serve a key role in cellular immunity (22-23).

Taken together, these data demonstrate that CTX is able to impede cellular immune function, and that FZQJ granules are able to prevent CTX-induced immune suppression in $\mathrm{H} 22$ tumor-bearing mice.

FZQJ exhibits no hepatic and renal toxicity. As presented in Table I, similar levels of ALT, BUN and CRE were observed between the vehicle group and the FZQJ group. CTX induced a marked increase of AST as predicted, and FZQJ was not able to prevent the CTX-induced deterioration of hepatic function. The results demonstrate that FZQJ was unable to alleviate CTX-induced hepatic injury.

\section{Discussion}

FZQJ has previously been used as adjuvant treatment during chemotherapy for gastrointestinal malignancies (24). The present study demonstrated that FZQJ is able to potentiate the anticancer efficacy of CTX, and prevent CTX-induced immune suppression and body weight loss without overt hepatorenal toxicity in $\mathrm{H} 22$ tumor-bearing mice. The underlying mechanisms for these processes may include FZQJ-induced tumor cell apoptosis and stimulated IL- 2 and TNF- $\alpha$ production to enhance cellular immune function.

Numerous studies have demonstrated that Fuzhegn Guben herbs and their ingredients are able to enhance the anticancer effects of chemotherapy and/or radiotherapy whilst reducing certain side effects $(5,25)$. For example, ginsenoside $\mathrm{Rg} 3$ combined with CTX decreased cell susceptibility to drug resistance and improved survival time in C57/BL6 mice with Lewis lung carcinoma (26). Shenqi Fuzheng injection has also been demonstrated to improve the immune function of patients with breast cancer receiving neoadjuvant chemotherapy (27). The present study observed that FZQJ produced an antineoplastic effect; however, this was less pronounced, compared with CTX. Notably, FZQJ was able to significantly potentiate the antineoplastic effect of CTX, with the absence of associated and overt side effects. The antineoplastic effect of FZQJ may be associated with the induction of $\mathrm{H} 22$ cell apoptosis. However the mechanism underlying the apoptosis-inducing action of FZQJ remains to be elucidated. Previous studies have demonstrated that FZQJ-induced hepatoma cell apoptosis occurs via the regulation of $\mathrm{Bcl}-2$ and Bax expression in vitro (13). In the present study, FZQJ was observed to stimulate IL-2 and TNF- $\alpha$ production. TNF- $\alpha$ is able to induce mitochondrial-mediated apoptosis via the activation of $\mathrm{Bcl}-2$ family proteins, reactive oxygen species, C-Jun, C-Jun $\mathrm{N}$ terminal kinases and cathepsin B (28-31). IL-2 is able to enhance the antitumor efficacy of TNF- $\alpha$, despite being less cytotoxic itself (32). In addition, Hedyotis diffusa Willd and Prunella vulgaris in FZQJ granules were reported to induce cell apoptosis via modulation of the IL-6/signal transducer and activator of transcription 3, MAPK and mitochondria-dependent signaling pathways $(33,34)$. The present and previous studies indicated that FZQJ granules were able to induce $\mathrm{H} 22$ cell apoptosis via IL-6/stat 3, MAPK and mitochondria-dependent pathway as well as stimulating IL-2 and TNF- $\alpha$ production.

The current study also observed fewer $\mathrm{CD} 3^{+} \mathrm{T}, \mathrm{CD} 4^{+} \mathrm{T}$ and NK cells in the peripheral blood of the vehicle group, compared with that of the blank group. The results indicated that cellular immune function was impaired once the blank mice were inoculated subcutaneously with $\mathrm{H} 22$ cells. Conversely, mice in the vehicle group exhibited a higher PLT count. This was in accordance with the thrombocytosis observed in patients with hepatic tumors (35). A possible underlying mechanism may be associated with tumor cell-stimulated production of thrombopoietin and IL-6, which promote PLT proliferation and activation $(35,36)$. When CTX alone was administrated for seven days continuously, the WBC, PLT, LY, CD4 ${ }^{+} \mathrm{T}$ helper, $\mathrm{CD}^{+} \mathrm{T}$ cytotoxic/suppressor, $\mathrm{CD}^{+} \mathrm{T}$ and $\mathrm{NK}$ cells, concentration of $\mathrm{Hb}$, thymus index and mouse body weight all decreased, indicating that CTX induced bone marrow suppression and a gastrointestinal reaction, which are common symptoms in patients receiving this treatment. The present 
study also demonstrated that FZQJ not only exhibited no toxicity, but that it also significantly increased the numbers of blood cells, the thymus index and body weight. Therefore, FZQJ may combat CTX-induced anemia and the decreased cellular immune function, as well as alleviate certain side effects of this treatment in the gastrointestinal tract.

Pharmacological studies have demonstrated that Astragalus membranaceus, Ligustrum lucidum, Ganoderma lucidum and Rhizoma dioscorea contain potent immune stimulants, for example polysaccharides, which trigger the production of numerous cytokines in vivo, including IL-2, IL-12 and TNF- $\alpha$, which may activate T and $\mathrm{B}$ cells (37-41) and modulate the balanced association between Th1 and Th 2 cytokines $(42,43)$. Concordantly, the present study demonstrated that FZQJ was able to upregulate the expression of IL- 2 and TNF- $\alpha$, which may be responsible for the observed increase in the $\mathrm{T}$ and NK cell counts. In a previous study, Astragalus membranaceus was able to increase serum megakaryocyte colony-stimulating activity and accelerate the recovery of hematopoiesis following bone marrow suppression in anemic mice, which may provide an explanation for the improvement of bone marrow suppression observed following FZQJ administration in CTX-treated mice (44). Finally, Hedyotis diffusa Willd, a component of FZQJ, was also reported to be capable of protecting the gastrointestinal mucosa (45), which may explain the observed improvement in CTX-induced body weight loss.

In conclusion, FZQJ not only improves the anticancer efficacy of CTX, but may also alleviate its adverse effects. Therefore, FZQJ may provide a promising adjuvant treatment during chemotherapy and/or radiotherapy for HCC.

\section{Acknowledgements}

This study was supported by the Natural Science Foundation of Fujian Province (grant nos. 2015J01689 and 2014J01421).

\section{References}

1. Bosch FX, Ribes J, Cléries R and Díaz M: Epidemiology of hepatocellular carcinoma. Clin Liver Dis 9: 191-211, v, 2005.

2. Tanaka M, Katayama F, Kato H, Tanaka H, Wang J, Qiao YL and Inoue M: Hepatitis B and C virus infection and hepatocellular carcinoma in China: A review of epidemiology and control measures. J Epidemiol 21: 401-416, 2011.

3. European Association for the Study of the Liver; European Organisation for Research and Treatment of Cancer: EASL-EORTC clinical practice guidelines: Management of hepatocellular carcinoma. J Hepatol 56: 908-943, 2012.

4. Wörns MA, Weinmann A, Schuchmann M and Galle PR: Systemic therapies in hepatocellular carcinoma. Dig Dis 27: $175-188,2009$

5. Konkimalla VB and Efferth T: Evidence-based Chinese medicine for cancer therapy. J Ethnopharmacol 116: 207-210, 2008.

6. Wang Z, Li J, Ji Y, An P, Zhang S and Li Z: Traditional herbal medicine: A review of potential of inhibitory hepatocellular carcinoma in basic research and clinical trial. Evid Based Complement Alternat Med 2013: 268963, 2013.

7. Zhang J and Shan BE: Recent advances on traditional Chinese medicine in immunoregulation and anticancer mechanism. Chin J Immunol 22: 385-388, 2006 (In Chinese).

8. Wu QX and Xu ZY: Advances on traditional Chinese medicine in myelosuppression. Zhejiang J Tradit Chin Med 45: 618-621, 2010 (In Chinese).

9. Shi Y, Wang $\mathrm{CH}$ and Gong XG: Apoptosis-inducing effects of two anthraquinones from Hedyotis diffusa WILLD. Biol Pharm Bull 31: 1075-1078, 2008
10. Lin SY, Shen CY, Jiang JP, Wu LQ, Dai TY, Qian WB and Meng HT: Apoptosis of multiple myeloid cells induced by polysaccharides extracts from Hedyotis diffusa and its mechanism. Zhonghua Xue Ye Xue Za Zhi 34: 337-340, 2013 (In Chinese).

11. Zhou QX, Liu F, Zhang JS, Lu JG, Gu ZL and Gu GX: Effects of triterpenic acid from Prunella vulgaris L. on glycemia and pancreas in rat model of streptozotozin diabetes. Chin Med J (Engl) 126: 1647-1653, 2013.

12. Si WK, Cheng A, Li P, Liu B, Gao LH and Yao J: Study on apoptosis of human hepatoma cell line HepG2 induced by matrine. Acta Academiae Medicinae Militaris Tertiae 23: 816-820, 2001 (In Chinese).

13. Chen XZ, Li JN, Zhang YQ, Cao ZY, Liu ZZ, Wang SQ, Liao LM and Du J: Fuzheng Qingjie recipe induces apoptosis in HepG2 cells via P38 MAPK activation and the mitochondria-dependent apoptotic pathway. Mol Med Rep 9: 2381-2387, 2014.

14. Lee CS and Gibson NW: DNA interstrand cross-links induced by the cyclopropylpyrroloindole antitumor agent bizelesin are reversible upon exposure to alkali. Biochemistry 32: 9108-9114, 1993.

15. Pisani F, Sciuto R, Dessanti ML, Giannarelli D, Kayal R, Rea S, Marchesi $\mathrm{F}$ and Marino M: Long term efficacy and safety of Fludarabine, Cyclophosphamide and Rituximab regimen followed by (90)Y-ibritumomab tiuxetan consolidation for the treatment of relapsed grades 1 and 2 follicular lymphoma. Exp Hematol Oncol 4: 17, 2015.

16. Fosså A, Muer M, Kasper C, Welt A, Seeber S and Nowrousian MR: Bolus vincristine and epirubicin with cyclophosphamide and dexamethasone (VECD) as induction and salvage treatment in multiple myeloma. Leukemia 12: 422-426, 1998.

17. Cain JM, Collins C, Petersdorf S, Figge DC, Tamimi HK, Greer BE and Livingston RB: Phase II study of high-dose cisplatin, etoposide, and cyclophosphamide for refractory ovarian cancer. Am J Obstet Gynecol 174: 1688-1694, 1996.

18. Tsujii H, Kamada T, Tsuji H, Takamura A, Matsuoka Y, Usubuchi $\mathrm{H}$ and Irie G: Improved results in the treatment of nasopharyngeal carcinoma using combined radiotherapy and chemotherapy. Cancer 63: 1668-1672, 1989.

19. Tang TC, Man S, Lee CR, Xu P and Kerbel RS: Impact of metronomic UFT/cyclophosphamide chemotherapy and antiangiogenic drug assessed in a new preclinical model of locally advanced orthotopic hepatocellular carcinoma. Neoplasia 12: 264-274, 2010.

20. Fraiser LH, Kanekal S and Kehrer JP: Cyclophosphamide toxicity. Characterising and avoiding the problem. Drugs 42: 781-795, 1991.

21. Lozano GM, Bejarano I, Espino J, González D, Ortiz A, García JF, Rodríguez AB and Pariente JA: Relationship between caspase activity and apoptotic markers in human sperm in response to hydrogen peroxide and progesterone. J Reprod Dev 55: 615-621, 2009.

22. Taniguchi T and Minami Y: The IL-2/IL-2 receptor system: A current overview. Cell 73: 5-8, 1993.

23. Locksley RM, Killeen $\mathrm{N}$ and Lenardo MJ: The TNF and TNF receptor superfamilies: Integrating mammalian biology. Cell 104: 487-501, 2001.

24. Cao ZY and Lan L: Experience of Professor Du Jian in fighting against gastrointestinal cancer by integrative therapy. J Fujian Univ Tradit Chin Med 21: 51-53, 2011 (In Chinese).

25. Qi F, Li A, Inagaki Y, Gao J, Li J, Kokudo N, Li XK and Tang W: Chinese herbal medicines as adjuvant treatment during chemo- or radio-therapy for cancer. Biosci Trends 4: 297-307, 2010

26. Zhang Q, Kang X and Zhao W: Antiangiogenic effect of low-dose cyclophosphamide combined with ginsenoside $\mathrm{Rg} 3$ on Lewis lung carcinoma. Biochem Biophys Res Commun 342: 824-828, 2006.

27. Dai Z, Wan X, Kang H, Ji Z, Liu L, Liu X, Song L, Min W and Ma X: Clinical effects of shenqi fuzheng injection in the neoadjuvant chemotherapy for local advanced breast cancer and the effects on T-lymphocyte subsets. J Tradit Chin Med 28: 34-38, 2008.

28. Yin XM and Ding WX: Death receptor activation-induced hepatocyte apoptosis and liver injury. Curr Mol Med 3: 491-508, 2003.

29. Nagai H, Matsumaru K, Feng G and Kaplowitz N: Reduced glutathione depletion causes necrosis and sensitization to tumor necrosis factor-alpha induced apoptosis in cultured mouse hepatocytes. Hepatology 36: 55-64, 2002.

30. Liu H, Lo CR and Czaja MJ: NF-kappaB inhibition sensitizes hepatocytes to TNF-induced apoptosis through a sustained activation of JNK and c-Jun. Hepatology 35: 772-778, 2002. 
31. Guicciardi ME, Deussing J, Miyoshi H, Bronk SF, Svingen PA, Peters C, Kaufmann SH and Gores GJ: Cathepsin B contributes to TNF-alpha-mediated hepatocyte apoptosis by promoting mitochondrial release of cytochrome c. J Clin Invest 106 1127-1137, 2000.

32. Owen-Schaub LB, Crump WL III, Morin GI and Grimm EA Regulation of lymphocyte tumor necrosis factor receptors by IL-2. J Immunol 143: 2236-2241, 1989.

33. Lin J, Li Q, Chen H, Lin H, Lai Z and Peng J: Hedyotis diffusa Willd. extract suppresses proliferation and induces apoptosis via IL-6-inducible STAT3 pathway inactivation in human colorectal cancer cells. Oncol Lett 9: 1962-1970, 2015.

34. Woo HJ, Jun do Y, Lee JY, Woo MH, Yang CH and Kim YH: Apoptogenic activity of $2 \alpha, 3 \alpha$-dihydroxyurs-12-ene-28-oic acid from Prunella vulgaris var. lilacina is mediated via mitochondria-dependent activation of caspase cascade regulated by Bcl-2 in human acute leukemia Jurkat T cells. J Ethnopharmacol 135: 626-635, 2011

35. Carrington PA, Carr TF, Stevens RF and Evans DI: Thrombocytosis associated with solid tumors in children. Pediatr Hematol Oncol 9: 289-291, 1992

36. Mantadakis E, Tsalkidis A and Chatzimichael A: Thrombocytosis in childhood. Indian Pediatr 45: 669-677, 2008.

37. Yang B, Xiao B and Sun T: Antitumor and immunomodulatory activity of Astragalus membranaceus polysaccharides in $\mathrm{H} 22$ tumor-bearing mice. Int J Biol Macromol 62: 287-290, 2013.

38. Sun Y, Hersh EM, Talpaz M, Lee SL, Wong W, Loo TL and Mavligit GM: Immune restoration and/or augmentation of loca graft versus host reaction by traditional Chinese medicinal herbs. Cancer 52: 70-73, 1983
39. Sun Y, Hersh EM, Lee SL, McLaughlin M, Loo TL and Mavligit GM: Preliminary observations on the effects of the Chinese medicinal herbs Astragalus membranaceus and Ligustrum lucidum on lymphocyte blastogenic responses. J Biol Response Mod 2: 227-237, 1983.

40. Yue GG, Chan BC, Han XQ, Cheng L, Wong EC, Leung PC, Fung KP, Ng MC, Fan K, Sze DM and Lau CB: Immunomodulatory activities of Ganoderma sinense polysaccharides in human immune cells. Nutr Cancer 65: 765-774, 2013.

41. Kohguchi M, Kunikata T, Watanabe H, Kudo N, Shibuya T, Ishihara T, Iwaki K, Ikeda M, Fukuda S and Kurimoto M: Immuno-potentiating effects of the antler-shaped fruiting body of Ganoderma lucidum (Rokkaku-Reishi). Biosci Biotechnol Biochem 68: 881-887, 2004.

42. Chen SM, Tsai YS, Lee SW, Liu YH, Liao SK, Chang WW and Tsai PJ: Astragalus membranaceus modulates Th1/2 immune balance and activates PPAR $\gamma$ in a murine asthma model. Biochem Cell Biol 92: 397-405, 2014.

43. Wang J, Shan A, Liu T, Zhang C and Zhang Z: In vitro immunomodulatory effects of an oleanolic acid-enriched extract of Ligustrum lucidum fruit (Ligustrum lucidum supercritical $\mathrm{CO} 2$ extract) on piglet immunocytes. Int Immunopharmacol 14: 758-763, 2012.

44. Zhu X and Zhu B: Effect of Astragalus membranaceus injection on megakaryocyte hematopoiesis in anemic mice. Hua Xi Yi Ke Da Xue Xue Bao 32: 590-592, 2001 (In Chinese).

45. Wang GY, Li ZB, Shi JX, Wang H, Gao QF, Gen LF, Zhu JJ and Zhao QS: Oldenlandia diffusa protects against indomethacin-induced injury of the gastrointestinal mucosa in rats. Hebei J Tradit Chin Med 23: 70-71, 2001 (In Chinese). 